\title{
Telomere-to-telomere assembly of the genome of an individual Oikopleura dioica from Okinawa using Nanopore-based sequencing
}

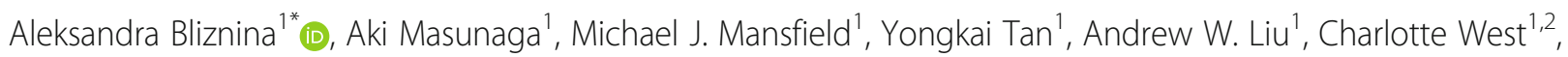
Tanmay Rustagi ${ }^{1}$, Hsiao-Chiao Chien ${ }^{1}$, Saurabh Kumar ${ }^{1}$, Julien Pichon ${ }^{1}$, Charles Plessy ${ }^{1 *}$ and

Nicholas M. Luscombe

\begin{abstract}
Background: The larvacean Oikopleura dioica is an abundant tunicate plankton with the smallest (65-70 Mbp) nonparasitic, non-extremophile animal genome identified to date. Currently, there are two genomes available for the Bergen (OdB3) and Osaka (OSKA2016) O. dioica laboratory strains. Both assemblies have full genome coverage and high sequence accuracy. However, a chromosome-scale assembly has not yet been achieved.

Results: Here, we present a chromosome-scale genome assembly (OKI2018_169) of the Okinawan O. dioica produced using long-read Nanopore and short-read Illumina sequencing data from a single male, combined with $\mathrm{Hi}-\mathrm{C}$ chromosomal conformation capture data for scaffolding. The OKI2018_169 assembly has a total length of $64.3 \mathrm{Mbp}$ distributed among 19 scaffolds. 99\% of the assembly is contained within five megabase-scale scaffolds. We found telomeres on both ends of the two largest scaffolds, which represent assemblies of two fully contiguous autosomal chromosomes. Each of the other three large scaffolds have telomeres at one end only and we propose that they correspond to sex chromosomes split into a pseudo-autosomal region and X-specific or Y-specific regions. Indeed, these five scaffolds mostly correspond to equivalent linkage groups in OdB3, suggesting overall agreement in chromosomal organization between the two populations. At a more detailed level, the OKI2018_169 assembly possesses similar genomic features in gene content and repetitive elements reported for OdB3. The Hi-C map suggests few reciprocal interactions between chromosome arms. At the sequence level, multiple genomic features such as GC content and repetitive elements are distributed differently along the short and long arms of the same chromosome.

* Correspondence: aleksandra.bliznina2@oist.jp; charles.plessy@oist.jp

'Genomics and Regulatory Systems Unit, Okinawa Institute of Science and Technology Graduate University, Okinawa, Japan

Full list of author information is available at the end of the article

(c) The Author(s). 2021 Open Access This article is licensed under a Creative Commons Attribution 4.0 International License, which permits use, sharing, adaptation, distribution and reproduction in any medium or format, as long as you give appropriate credit to the original author(s) and the source, provide a link to the Creative Commons licence, and indicate if changes were made. The images or other third party material in this article are included in the article's Creative Commons licence, unless indicated otherwise in a credit line to the material. If material is not included in the article's Creative Commons licence and your intended use is not permitted by statutory regulation or exceeds the permitted use, you will need to obtain permission directly from the copyright holder. To view a copy of this licence, visit http://creativecommons.org/licenses/by/4.0/ The Creative Commons Public Domain Dedication waiver (http://creativecommons.org/publicdomain/zero/1.0/) applies to the data made available in this article, unless otherwise stated in a credit line to the data. 
(Continued from previous page)

Conclusions: We show that a hybrid approach of integrating multiple sequencing technologies with chromosome conformation information results in an accurate de novo chromosome-scale assembly of O. dioica's highly polymorphic genome. This genome assembly opens up the possibility of cross-genome comparison between O. dioica populations, as well as of studies of chromosomal evolution in this lineage.

Keywords: Oikopleura dioica, Oxford Nanopore sequencing, Hi-C, Telomere-to-telomere, Chromosome-scale assembly, single individual

\section{Background}

Larvaceans (synonym: appendicularians) are among the most abundant and ubiquitous taxonomic groups within animal plankton communities $[1,2]$. They live inside self-built "houses" which are used to trap food particles [3]. The animals regularly replace houses as filters become damaged or clogged and a proportion of discarded houses with trapped materials eventually sink to the ocean floor. As such larvaceans play a significant role in global vertical carbon flux [4].

Oikopleura dioica is the best documented species among larvaceans. It possesses several invaluable features as an experimental model organism. It is abundant in coastal waters and can be easily collected from the shore. Multigenerational culturing is possible [5]. It has a short lifecycle of 4 days at $23^{\circ} \mathrm{C}$ and remains freeswimming throughout its life [6]. As a member of the tunicates, a sister taxonomic group to vertebrates, $O$. dioica offers insights into their evolution [7].

O. dioica's genome size is $65-70 \mathrm{Mbp}[8,9]$, making it one of the smallest among all sequenced animals. Interestingly, genome-sequencing of other larvacean species uncovered large variations in genome sizes, which correlated with the expansion of repeat families [10]. $O$. dioica is distinguished from other larvaceans as the only reported dioecious species [11] with sex determination system using an X/Y pair of chromosomes [9]. The first published genome assembly of $O$. dioica (OdB3, B stands for Bergen) was performed with Sanger sequencing which allowed for high sequence accuracy but limited coverage [9]. The OdB3 assembly was scaffolded with a physical map produced from BAC-end sequences, which revealed two autosomal linkage groups and a sex chromosome with a long pseudo-autosomal region (PAR) [9]. Recently, a genome assembly for a mainland Japanese population of O. dioica (OSKA2016, OSKA denotes Osaka) was published, which displayed a high level of coding sequence divergence compared with the OdB3 reference [12, 13]. Although OSKA2016 was sequenced with single-molecule long reads produced with the PacBio RSII technology, it does not have chromosomal resolution.

Historical attempts at karyotyping $O$. dioica by traditional histochemical stains arrived at different chromosome counts, ranging between $n=3$ [14] and $n=$ 8 [15]. In preparation for this study, we karyotyped the Okinawan $O$. dioica by staining centromeres with antibodies targeting phosphorylated histone H3 serine 28 [16], and determined a count of $n=3$. This is also in agreement with the physical map of OdB3 [9].

Currently, the method of choice for producing chromosome-scale sequences is to assemble contigs using long reads ( $10 \mathrm{~kb}$ or more) produced by either the Oxford Nanopore or PacBio platforms, and to scaffold them using $\mathrm{Hi}-\mathrm{C}$ contact maps $[17,18]$. To date, there have been no studies of chromosome contacts in Oikopleura or any other larvaceans.

Here, we present a chromosome-length assembly of the Okinawan $O$. dioica genome sequence generated with datasets stemming from multiple genomic technologies and data types, namely long-read sequencing data from Oxford Nanopore, short-read sequences from Illumina and $\mathrm{Hi}-\mathrm{C}$ chromosomal contact maps (Fig. 1).

\section{Results}

\section{Genome sequencing and assembly}

O. dioica's genome is highly polymorphic [9], making assembly of its complete sequence challenging. To reduce the level of variation, we sequenced genomic DNA from a single $O$. dioica male. The low amount of extracted DNA is an issue when working with small-size organisms like $O$. dioica. Therefore, we optimized the extraction and sequencing protocols to allow for low-template input DNA yields of around $200 \mathrm{ng}$ and applied a hybrid sequencing approach using Oxford Nanopore reads to span repeat-rich regions and Illumina reads to correct individual nucleotide errors. The Nanopore run gave 8.2 million reads $(221 \times$ coverage) with a median length of $840 \mathrm{bp}$ and maximum length of $166 \mathrm{~kb}$ (Fig. 2a). Based on k-mer counting of the Illumina reads, the genome was estimated to contain $\sim 50 \mathrm{Mbp}$ (Fig. 2b) - comparable in size to the OdB3 and OSKA2016 assemblies and a relatively high heterozygosity of $\sim 3.6 \%$. We used the Canu pipeline [19] to correct, trim and assemble Nanopore reads, yielding a draft assembly comprising 175 contigs with a weighted median N50 length of 3.2 Mbp. We corrected sequencing errors and local misassemblies of the draft contigs with Nanopore reads using 


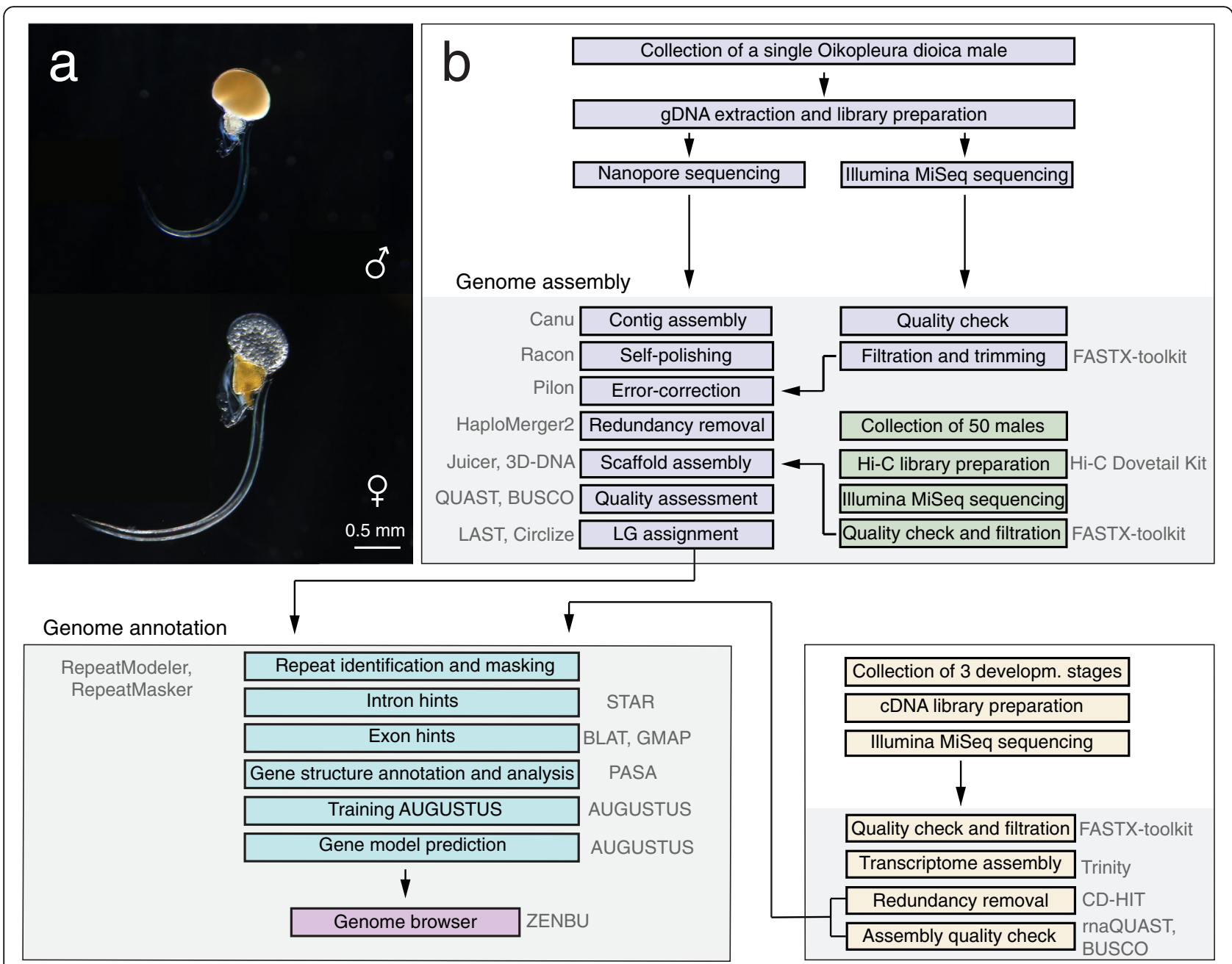

Fig. 1 Genome assembly and annotation workflow used to generate the OKI2018_I69 genome assembly. a Life images of adult male (top) and female (bottom) O. dioica. b The assembly was generated using Nanopore and Illumina data, followed by scaffolding using Hi-C chromosomal capture information data

Racon, and then with Illumina reads using Pilon. The initial Okinawa O. dioica assembly length was $99.3 \mathrm{Mbp}$, or $~ 1.5$ times longer than the OdB3 genome at 70.4 Mbp. Merging haplotypes with HaploMerger2 resulted in two sub-assemblies (reference and allelic) of 64.3 Mbp with an N50 of $4.7 \mathrm{Mbp}$. Repeating the procedure on a second individual from the same culture showed overall agreement in assembly lengths, sequences and structures (Fig. 2c).

To scaffold the genome, we sequenced Hi-C libraries from a pool of $\sim 50$ individuals from the same culture. More than $99 \%$ of the $\mathrm{Hi}-\mathrm{C}$ reads could be mapped to the contig assembly. After removing duplicates, Hi-C contacts were passed to the 3D-DNA pipeline to correct major misassemblies, as well as order and orient the contigs. The resulting assembly consisted of 8 megabase-scale scaffolds containing $99 \%$ of the total sequence (Fig. 3a), and 14 smaller scaffolds that account for the remaining $663 \mathrm{kbp}$ (lengths ranging from 2.9 to $131.6 \mathrm{kbp}$ ). One of the small scaffolds is a draft assembly of the mitochondrial genome that we discuss below. Most of the other smaller scaffolds are highly repetitive and might represent unplaced fragments of centromeric or telomeric regions. We annotated telomeres by searching for the TTAGGG repeat sequence and found that most of the megabase-scale scaffolds have single telomeric regions: therefore, we reasoned that they represent chromosome arms. Indeed, pairwise genome alignment to OdB3 identified two syntenic scaffolds for each autosomal linkage group, two for the pseudo-autosomal region (PAR) and one for each sex-specific region. Since we had previously inferred a karyotype of $n=3$ by immunohistochemistry [16], we completed the assembly by pairing the megabase-scale scaffolds into chromosome arms based on their synteny with the OdB3 physical map (Fig. 3b). The final assembly named OKI2018_I69 


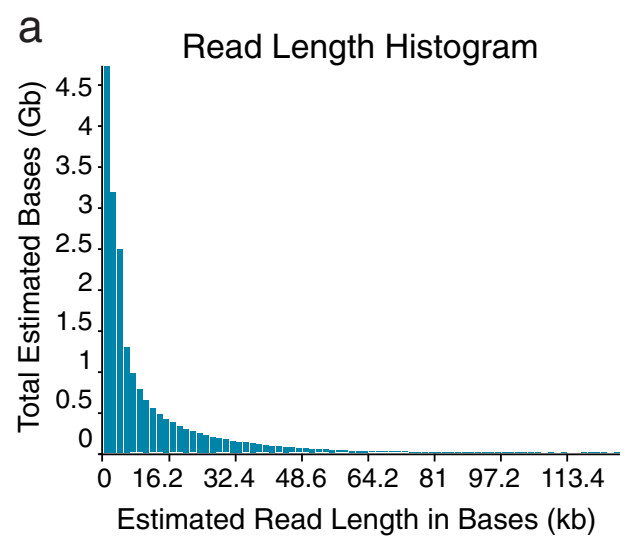

b

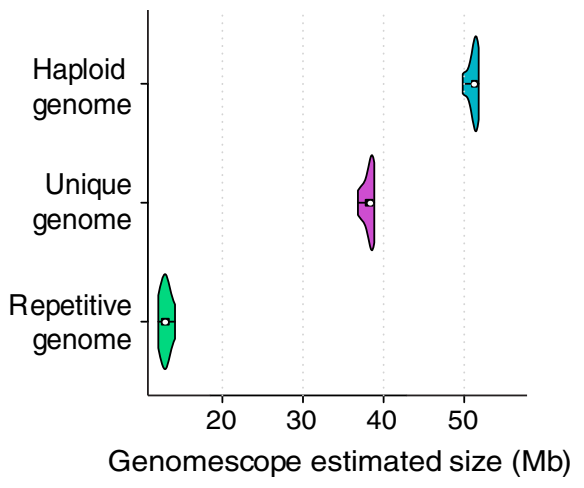

C

169 contigs

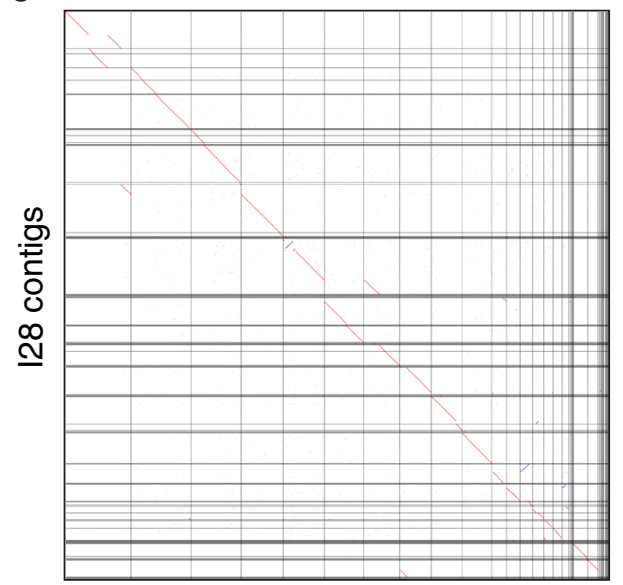

Fig. 2 Quality control checks implemented on different steps of genome sequencing and assembly. a Graph showing length distribution of raw Nanopore reads used to generate the OKI2018_169 assembly. b Estimated total and repetitive genome size based on $k$-mer counting of the Illumina paired-end reads used for polishing the OKI2018_I69 assembly. c Pairwise genome alignment of the contig assemblies of 169 and I28 0. dioica individuals

(Table 1; Suppl. Table 1) comprises telomere-totelomere assemblies of the autosomal chromosomes 1 (chr 1) and 2 (chr 2). The sex chromosomes are split into pseudo-autosomal region (PAR) and X-specific region (XSR) or Y-specific region (YSR; Fig. 3). We assume that the sex-specific regions belong to the long arm of the PAR, as the long arm does not contain any telomeric repeats (Fig. 4a). Alignment of the Illumina polishing reads to the OKI2018_I69 assembly estimated an error rate of $1.3 \%$ showing high sequence accuracy.

The genome-wide contact matrix from the $\mathrm{Hi}-\mathrm{C}$ data (Fig. 3c) shows bright, off-diagonal spots that suggest spatial clustering of the telomeres and centromeres both within the same and across different chromosomes [18]. The three centromeric regions are outside the sexspecific regions, dividing the PAR and both autosomes into long and short arms. The two sex-specific regions have lower apparent contact frequencies compared with the rest of the assembly which is consistent with their haploid status in males. The chromosome arms themselves show few interactions between each other, even when they are part of the same chromosome.

\section{Chromosome-level features}

The genome contains between 1.4 and 2.6 Mbp of tandem repeats (detected using the tantan and ULTRA algorithms respectively with maximum period lengths of 100 and 2000). Subtelomeric regions tend to contain retrotransposons or tandem repeats with longer periods. We also found telomeric repeats in smaller scaffolds. A possible explanation is that subtelomeric regions display high heterozygosity, leading to duplicated regions that fail to assemble with the chromosomes. Alternatively, these scaffolds could be peri-centromeric regions containing interstitial telomeric sequences. In some species, high-copy tandem repeats can be utilized to discover the position of centromeric regions [20]; however, we could not find such regions. Additional experimental 


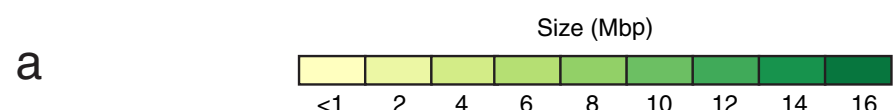

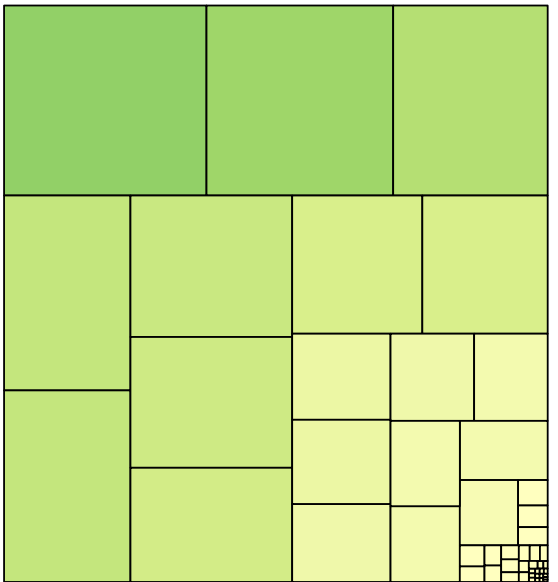

OKI2018_I69 contigs

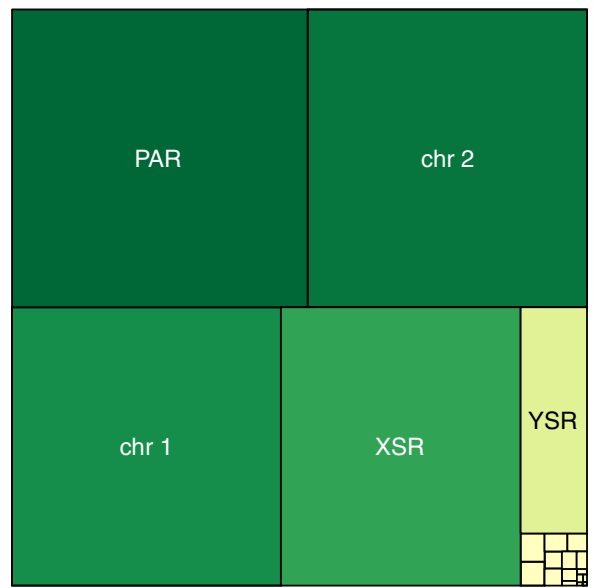

OKI2018_169

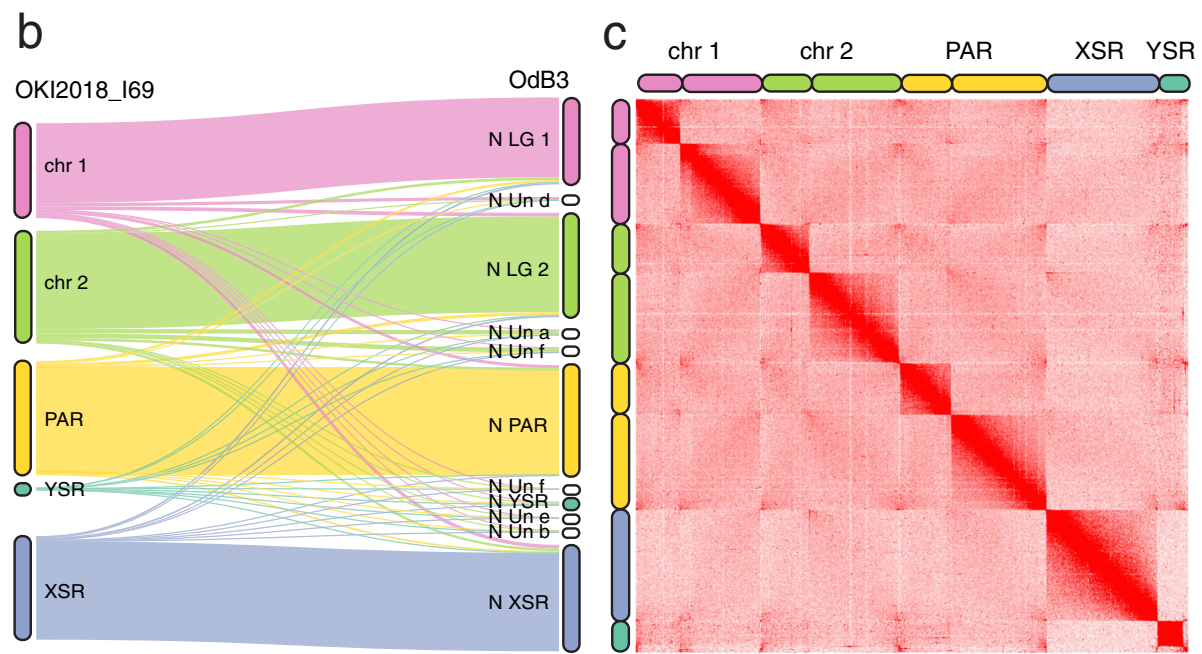

Fig. 3 OKI2018_I69 assembly of the Okinawan O. dioica. a Treemap comparison between the contig (left) and scaffold (right) assemblies of the O. dioica genome. Each rectangle represents a contig or a scaffold in the assembly with the area proportional to its length. b Comparison between the OKI2018_169 (left) and OdB3 (right) linkage groups. The Sankey plot shows what proportion of each chromosome in the OKI2018_169 genome is aligned to the OdB3 linkage groups. c Contact matrix generated by aligning Hi-C data set to the OKI2018_169 assembly with Juicer and 3D-DNA pipelines. Pixel intensity in the contact matrices indicates how often a pair of loci collocate in the nucleus

Table 1 Comparison of the OKI2018_169 assembly with the previously published O. dioica genomes

\begin{tabular}{llll}
\hline & OdB3 & OSKA2016 & OKI2018_169 \\
\hline Geographical origin & Bergen, Norway (North Atlantic) & Hyogo, Japan (Western Pacific) & Okinawa, Japan (Ryukyu archipelago) \\
Assembly length (Mbp) & 70.4 & 65.6 & 64.3 \\
Number of scaffolds & 1260 & 576 & 19 \\
Longest scaffold (Mbp) & 3.2 & 6.8 & 17.1 \\
Scaffold N50 (Mbp) & 0.4 & 1.5 & 16.2 \\
Number of contigs & 5917 & 746 & 42 \\
Contig N50 (Mbp) & 0.02 & 0.6 & 4.7 \\
GC content (\%) & 39.77 & 41.34 & 41.06 \\
Gap rate (\%) & 5.589 & 0.585 & 0.034 \\
Complete BUSCOs (\%) & 70.8 & 71.7 & 73.01 \\
\hline
\end{tabular}




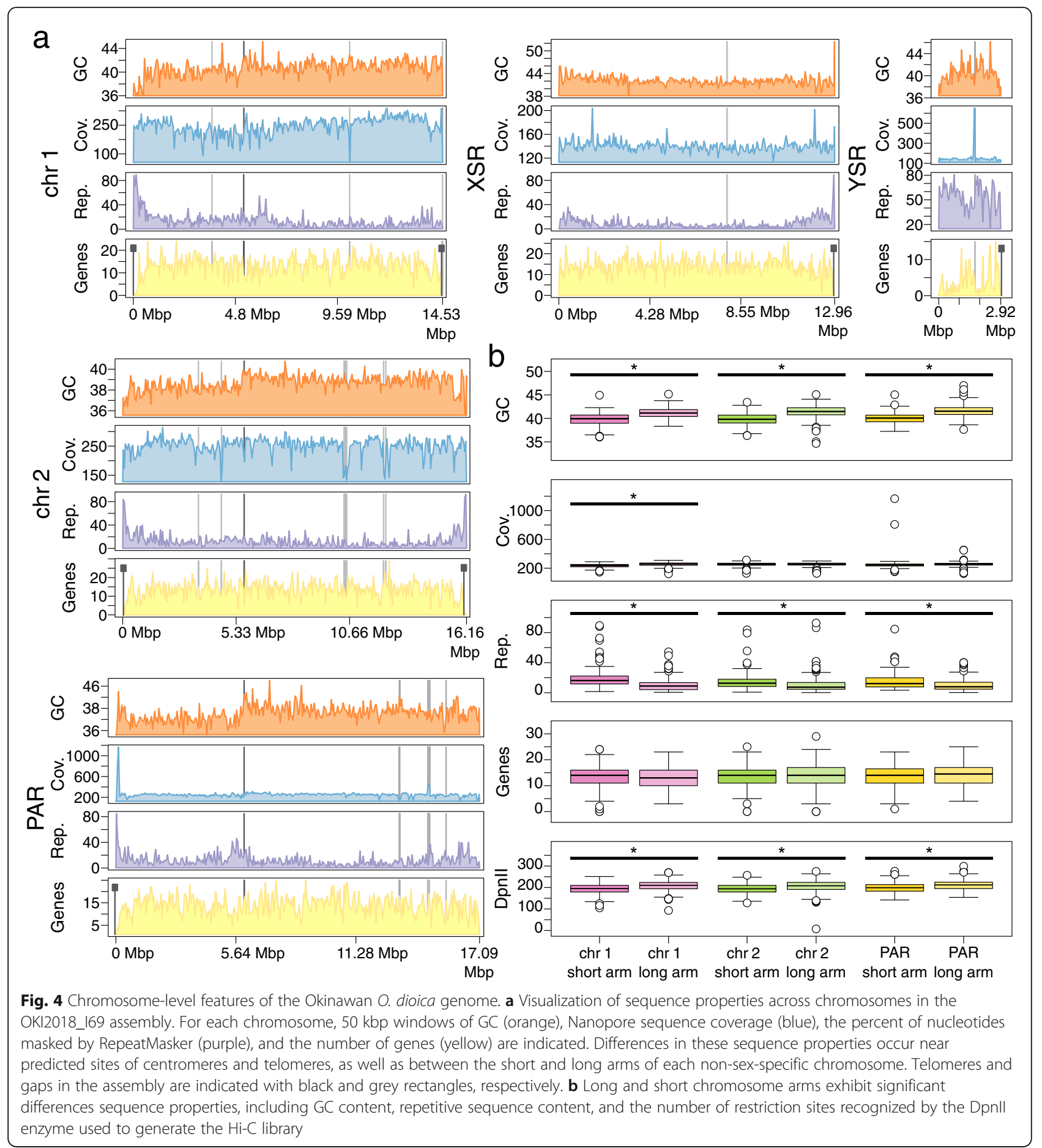

techniques such as chromatin immunoprecipitation and sequencing with centromeric markers might be necessary to resolve the centromeres precisely. Therefore, the current assembly skips over centromeric regions, represented as gaps of arbitrary size of $500 \mathrm{bp}$ in the chromosomal scaffolds.

We studied genome-scale features by visualizing them along whole chromosomes, from the short to long arm, centered on their centromeric regions. Most strikingly, there is a clear difference in sequence content between chromosome arms (Fig. 4; Supp. Table 3). The short arms consistently display depleted GC content and elevated repetitive content compared with the corresponding long arms. Although GC content tends to be weakly negatively correlated with repeat content, it is not currently possible to ascertain causality and the mechanism 
behind the marked difference in sequence content between the short and long chromosome arms remains unknown. It should be noted that the differences in GC contents affects the density of the GATC DpnII restriction enzyme recognition sites used for Hi-C library preparation; however, this bias is insufficient to explain the low degree of intra-chromosomal interaction observed in the $\mathrm{Hi}-\mathrm{C}$ contact maps.

\section{Quality assessment using BUSCO}

To assess the completeness of our assembly, we searched for 978 metazoan Benchmarking Universal Single-Copy Orthologs (BUSCOs) provided with the BUSCO tool [21-23]. To increase sensitivity, we trained BUSCO's gene prediction tool, AUGUSTUS [24], with transcript models generated from RNA-Seq data collected from the same laboratory culture (see below). We detected $73.0 \%$ of BUSCOs (Table 1), which is similar to OdB3 and OSKA2016 (Fig. 5a; Suppl. Table 4). All detected BUSCOs except one reside on the chromosomal scaffolds. As the reported fraction of detected genes is lower than for other tunicates such as Ciona intestinalis HT (94.6\%) [25] or Botrylloides leachii (89\%) [26], we searched for BUSCO genes in the transcriptomic training data (83.0\% present) and confirmed the presence of all but one by aligning the transcript sequence to the genome. We then inspected the list of BUSCO genes that were found neither in the genome nor in the transcriptome. Bibliographic analysis confirmed that BUSCO genes related to the peroxisome were lost from $\mathrm{O}$. dioica $[27,28]$. There are two possible explanations for the remaining missing genes: first is that protein sequence divergence [29] or length reduction [30] in Oikopleura complicate detection by BUSCO, and second is gene loss. In line with the possibility of gene loss, most BUSCO genes missing from our assembly are also undetectable in OdB3 and OSKA2016 (Fig. 5b; Suppl. Table 5). To summarize, the Okinawa assembly achieved comparable detection of universal single-copy conserved orthologs compared with previous $O$. dioica assemblies, and consistently undetectable genes may have been lost or diverged extensively in Oikopleura.

\section{Repeat annotation}

In order to identify repetitive elements in the OKI2018 I69 genome, we combined the results of several de novo repeat detection algorithms and used this custom library as an input to RepeatMasker to identify repeat sequences. Interspersed repeats make up $14.4 \%$ of the assembly (9.25 Mbp; Fig. 6), comparable to the $15 \%$ reported for OdB3 [9]. Of the annotated elements, the most abundant type is the long terminal repeat (LTRs; $\sim 4.6 \%$ ) with Ty3/gypsy Oikopleura transposons (TORs) dominating 2.97 Mbp of the sequence. Short interspersed nuclear elements (SINEs) make up a smaller portion of the OKI2018_I69 sequence $(<0.1 \%)$ compared with the OdB3 $(0.6 \%)$. It has been suggested that SINEs contribute significantly to genome size variation in other oikopleurids [10], but further analysis is required to determine whether that is the case at shorter evolutionary distances. Non-LTR LINE/Odin and Penelope-like elements are large components of most oikopleurid genomes [10], but they are almost absent from the OKI2018_I69 assembly. Indeed, 44\% of the predicted repeats in the Okinawan $O$. dioica could not be classified through searches against repeat databases and may either represent highly divergent relatives of known repeat classes, or novel repeats specific to Okinawan $O$. dioica.

\section{Gene annotation}

We annotated the OKI2018_I69 assembly using RNASeq-based gene prediction. RNA-Seq reads mapped to the assembly showed $99.14 \%$ agreement between the genome and transcriptome indicating high sequence accuracy. Annotation of the genome yielded 18,794 transcript isoforms distributed among 17,260 protein-coding genes. The number of predicted genes for the OKI2018 I69 is slightly lower than what was reported for OdB3 $(18,020)$ [9] and OSKA2016 (18,743) [13] (Table 2). The rest of the genes are either lost from the Okinawan $O$. dioica genome or were not assembled and/or annotated with our pipeline. On the other hand, the higher number of genes might be artifacts of the OdB3 and OSKA2016 annotations. The completeness of the annotation compares to the genome: BUSCO recovered $75.3 \%$ complete and $4.8 \%$ fragmented metazoan genes (Fig. 5a). Like the OdB3 assembly, gene density is very high at one gene per $3.7 \mathrm{kbp}$. OKI2018_I69 has similar gene and exon length distributions, and very short introns with a median length of only $49 \mathrm{bp}$ (Table 2). Indeed, we found a high frequency of the non-canonical (non-GT/AG) introns in the OKI2018_I69 (11\%). Previously, Denoeud et al. reported that $12 \%$ of the introns were noncanonical in the OdB3 [9]. Some of those non-canonical introns were found in the same genes as in the OdB3. However, more close examination is required to understand if it is the case for the rest of the genes. Therefore, overall genomic features seem to be conserved among O. dioica population despite large geographic distance.

The ribosomal DNA gene encoding the precursor of the $18 \mathrm{~S}, 5.8 \mathrm{~S}$ and $28 \mathrm{~S}$ rRNAs occurs as long tandem repeats that form specific chromatin domains in the nucleolus. We identified 4 full tandem copies of the rDNA gene at the tip of the PAR's short arm, separated by $8738 \mathrm{bp}$ (median distance). As this region has excess coverage of raw reads, and assemblies of tandem repeats are limited by the read length ( $99 \%$ of Nanopore reads in our data are shorter than $42,842 \mathrm{bp}$ ), we estimate that 


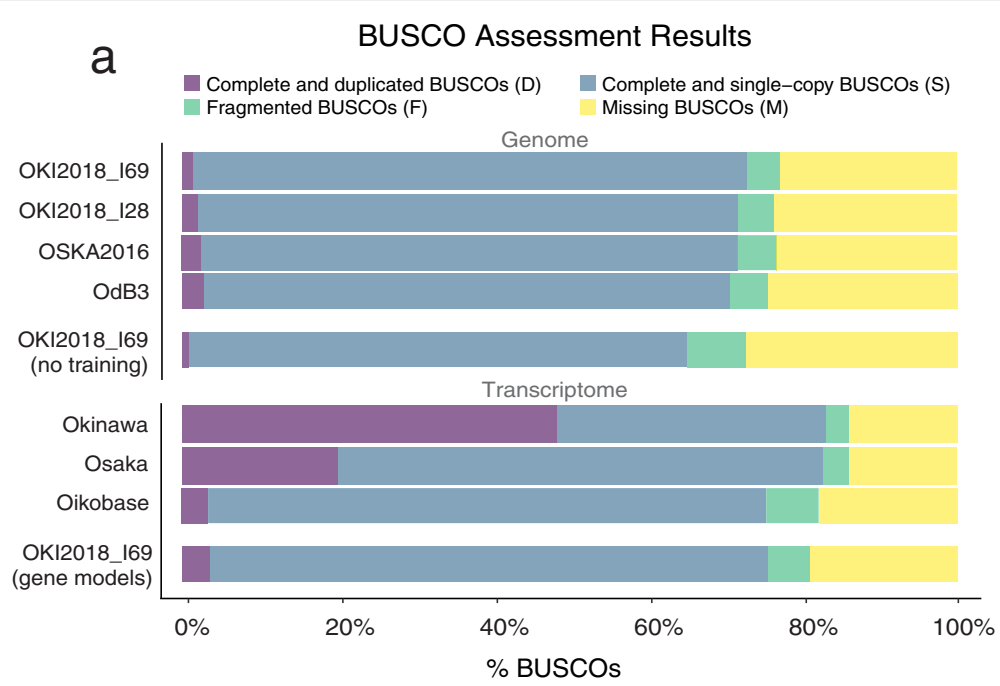

b

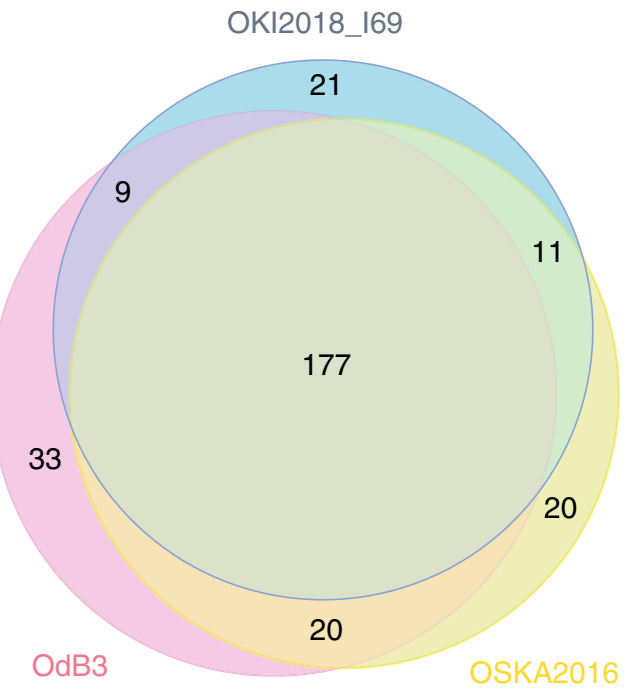

Fig. 5 Quality assessment of the OKI2018_169 genome assembly. a Proportion of BUSCO genes detected or missed in Oikopleura genomes and transcriptomes. The search on the OKI2018_169 assembly was repeated with default parameters ("no training") to display the effect of AUGUSTUS training. $\mathbf{b}$ Venn diagram showing the number of BUSCO genes missing in OKI2018_169, OdB3 and/or OSKA2016 genomes

the real number of the tandem rDNA copies could range between 20 (MiSeq) and 100 (Nanopore) copies. Between or flanking the rDNA genes, we also found short tandem repeats made of two to three copies of a 96-bp sequence. This tandem repeat is unique to the rDNA genes and to our reference and draft genomes, and was not found in the OdB3 reference nor in other larvacean genomes. The 5S rRNA is transcribed from loci distinct to the rDNA gene tandem arrays. In Oikopleura, they have the particularity of being frequently associated with the spliced leader (SL) gene and to form inverted repeats present in more than 40 copies [31]. We found 27 copies of these genes on every chromosomal scaffold except YSR, 22 of which were arranged in inverted tandem repeats. Altogether, we found in our reference genome one rDNA gene repeat region assembled at the end of a chromosome short arm. This sequence might provide useful markers for phylogenetic studies in the future.

\section{Draft mitochondrial genome scaffold}

We identified a draft mitochondrial genome among the smaller scaffolds, chrUn_12, by searching for mitochondrial sequences using the Cox1 protein sequence and the ascidian mitochondrial genetic code [32]. Automated annotation of this scaffold using the MITOS2 server detected the coding genes cob, cox1, nad1, cox3, nad4, cox2, and atp6 (Fig. 7a), which are the same as in Denoeud et al., 2010 [9] except for the $n d 5$ gene that is missing from our assembly. The open reading frames are often interrupted by T-rich regions, in line with 


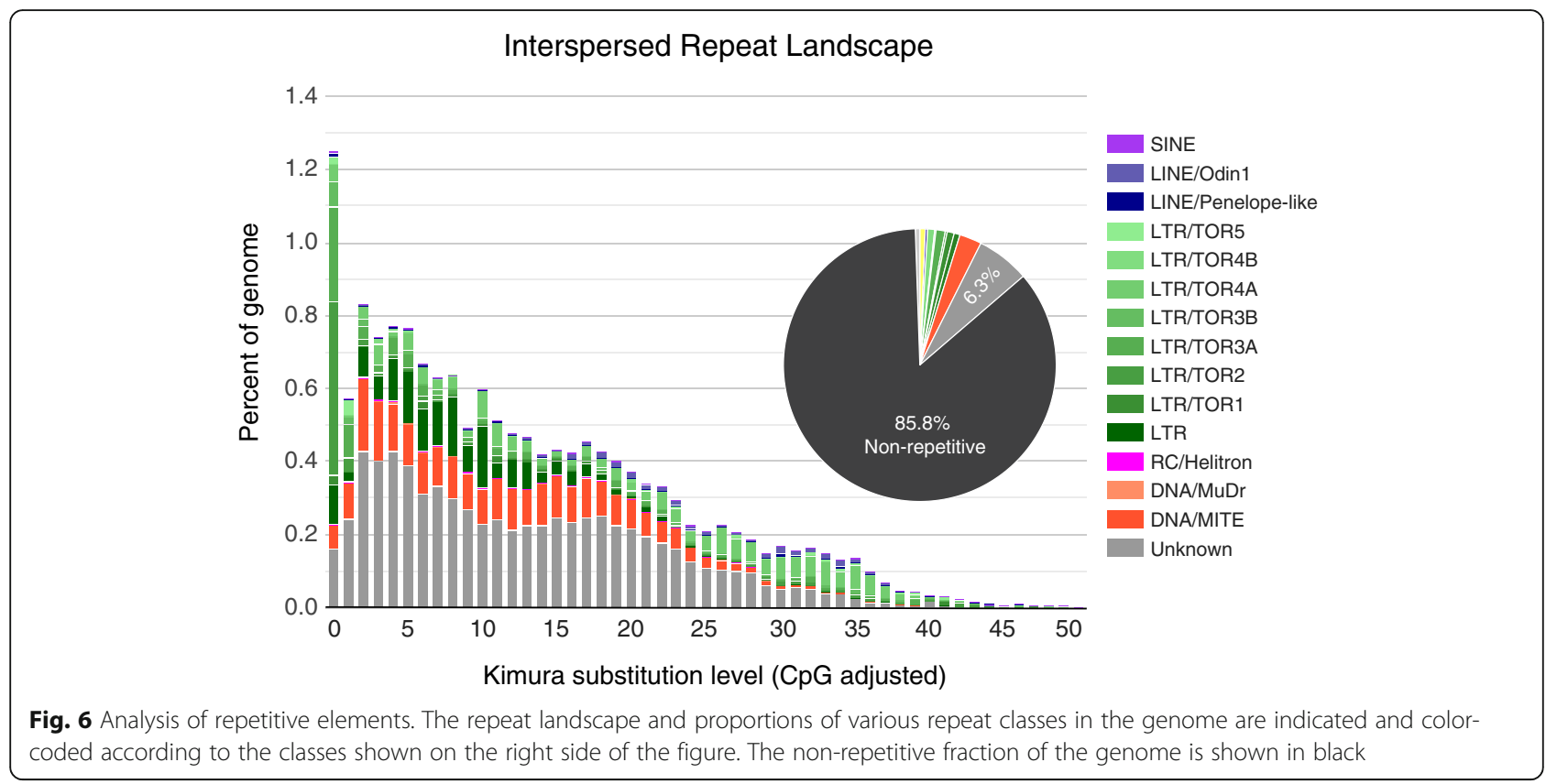

Denoeud et al. (2010) [9]. However, we cannot rule out the possibility that these regions represent sequencing errors, as homopolymers are difficult to resolve with the Nanopore technology available in 2019. The cob gene is interrupted by a long non-coding region, but this might be a missassembly. Indeed, an independent assembly using the flye software [33] with the --meta option to account for differential coverage also produced a draft mitochondrial genome, but its non-coding region was 2 kbp longer. Moreover, a wordmatch dotplot shows tandem repeats in this region (Fig. 7b), and thus this region is prone to assembly errors, especially with respect to the number of repeats. Altogether, the draft contig produced in our assembly shows as a proof of principle that sequencing reads covering the mitochondrial genome alongside the nuclear genome can be produced from a single individual, although it may need supporting data such as targeted resequencing in order to be properly assembled.

Table 2 Comparison of the annotations of the three O. dioica genome assemblies

\begin{tabular}{llll}
\hline & OdB3 & OSKA2016 & OKI2018_169 \\
\hline Masked sequence (\%) & 15.0 & - & 14.4 \\
Number of genes & 18,020 & 18,743 & 17,260 \\
Median gene length (bp) & 1488 & 1483 & 1505 \\
Median exon length (bp) & 159 & 155 & 152 \\
Median intron length & 48 & 51 & 49 \\
\hline
\end{tabular}

\section{Discussion}

\section{OKI2018_169 assembly quality}

Previously, different techniques have been used to sequence and assemble $O$. dioica genomes which have produced assemblies of varying quality. The Sangerbased OdB3 sequence was published in 2010 [9]. Due to limitations in sequencing technologies at the time, it is highly fragmented, comprising 1260 scaffolds with an N50 of $0.4 \mathrm{Mbp}$. The recently released OSKA2016 assembly was generated from long-read PacBio data and, therefore, has a larger N50 and fewer scaffolds (Table 1) [13]. Both assemblies have high sequence quality and nearly full genome coverage, but neither of them contains resolved chromosomes. However, Denoeud et al. (2010) [9] released a physical map calculated for OdB3 from $\mathrm{BAC}$ end sequences that comprises five linkage groups (LGs): two autosomal LGs, one pseudoautosomal region of sex chromosomes, and two sex specific regions ( $\mathrm{X}$ and $\mathrm{Y})$.

The use of reference chromosome information from a closely related species to order contigs or scaffolds into chromosome-length sequences is a common way to generate final genome assemblies [34]. However, this approach precludes discovery of structural variants. In our study, we first assembled long Nanopore reads de novo into contigs that we ordered and joined into megabasescale scaffolds using long-range $\mathrm{Hi}-\mathrm{C}$ data. The syntenybased approach with OdB3's linkage groups as a reference was only required to guide final pairing of chromosome arms into single scaffolds of chr 1, chr 2 and PAR, as we found that these scaffolds mostly align to one of the autosomal LGs or PAR. Therefore, any potential 


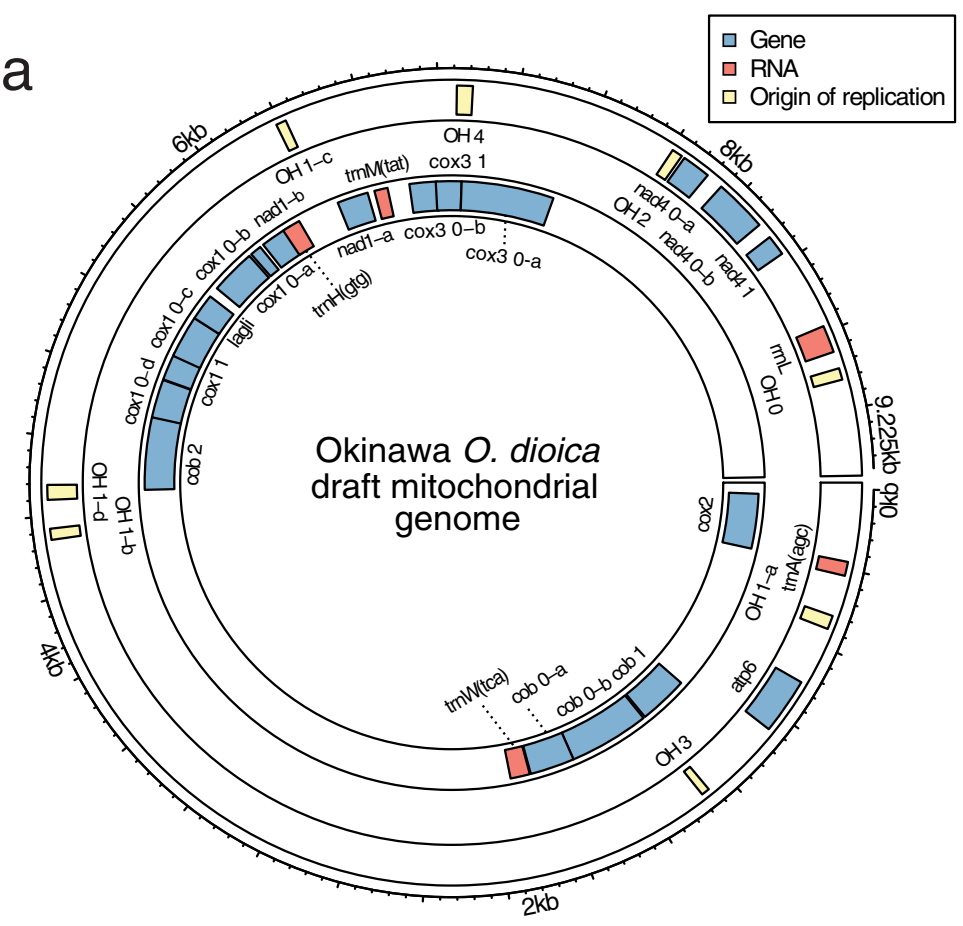

$\mathrm{b}$

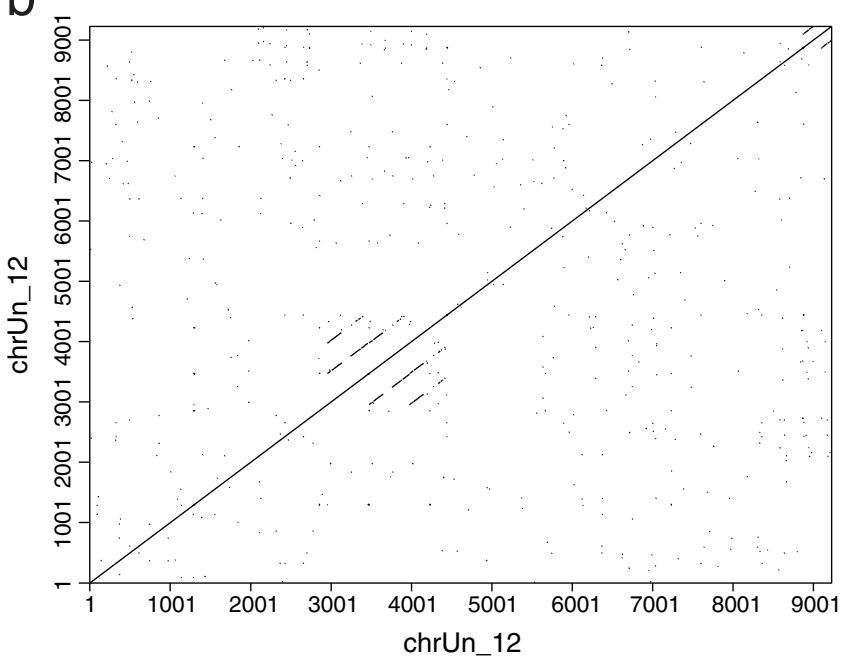

Fig. 7 Draft scaffold of the mitochondrial genome in the OKI2018_169 assembly. a Predicted gene annotation of the draft mitochondrial genome sequence. $\mathbf{b}$ Self-similarity plot of the draft mitochondrial genome sequence. A tandem repeat can be seen, which complicates the complete assembly of the mitochondrial genome from whole-genome sequencing data

assembly errors in OdB3 would not be transferred to our assembly. Apart from these syntenic relationships, our karyotyping results and the count of three centromeres on the $\mathrm{Hi}-\mathrm{C}$ contact map supports the presence of three pairs of chromosomes in the Okinawan $O$. dioica. However, there is a possibility that chromosome arms might have been exchanged between chromosomes in the Okinawan population. Additional experimental evidence is needed to confirm the pairing of chromosome arms, such as data generated by the Omni-C method which does not rely on restriction enzyme fragmentation.

Our synteny-based scaffolding is based on the simplest definition of synteny meaning "on the same chromosome". It does not make assumptions on gene order, which is why we report our results with a positionindependent Sankey plot in Fig. 3b. We initially assumed that animals collected from the Atlantic and Pacific oceans are from the same species and conserve these chromosomal properties. However, there are visible 
differences in gene number, gene order and repeat content compared with the OdB3 and OSKA2016. O. dioica is distributed all over the world, and all the populations are classified as a single species owing to the lack of obvious morphological differences and limited understanding of population structure. However, the short life span of $O$. dioica combined with limited mobility and high mutation rate contribute to an accelerated genome evolution that might have led to multiple speciation events. Sequence polymorphism was previously noted when comparing the OdB3 genome to genomic libraries of a laboratory strain collected on the North American Pacific coast [9], and more recently when comparing OdB3 to OSKA2016 [12, 13]. The chromosome-scale OKI2018_I69 assembly opens up the possibility for further work on cross-comparison among $O$. dioica populations that will elucidate the relation of the Okinawan populations to the North Atlantic and North Pacific ones.

\section{Inter-arm contacts}

The sequence of $O$. dioica's chromosomes and their contact map suggest that chromosome arms may be the fundamental unit of synteny in larvaceans. Hi-C contact matrices in vertebrates typically display greater intrachromosomal than inter-chromosomal interactions. A similar pattern was reported in the tunicate Ciona robusta (also known as intestinalis type A) [25] and the lancelet Branchiostoma floridae [35]. By comparison, in flies and mosquitoes, the degree of contacts between two arms of the same chromosome appear to be reduced but nonetheless more frequent than between different chromosomes [18]. Indeed, in Drosophila, the chromosome arms - which are termed Muller elements owing to studies with classical genetics [36] - are frequently exchanged between chromosomes across speciation events. $O$. dioica's genome shares with fruit flies its small size and small number of chromosomes. However, small chromosome size is also seen in the tunicate Ciona robusta, which has 14 meta- or sub-meta-centric pairs [37], with an average length of $\sim 8 \mathrm{Mbp}$ [25] that exhibit a more extensive degree of contacts, particularly for intra-chromosomal interactions across the centromeres [25]. As we prepared our $\mathrm{Hi}-\mathrm{C}$ libraries from adult animals, where polyploidy is high [38], we cannot rule out that it could be a possible cause of the low inter-arm interactions in our contact matrix. Further studies such as investigations of other developmental stages will be needed to elucidate the mechanism at work for the similarity between $O$. dioica and insect's chromosome contact maps.

\section{Visualization and access}

We prepared a public view of our reference genome in the ZENBU browser [39], displaying tracks for our gene models, in silico-predicted features such as repeats and non-coding RNAs, or syntenies with other Oikopleura genomes. To facilitate the study of known genes, we screened the literature for published sequences (Suppl. Table 6) and mapped them to the genome with a translated alignment. The ZENBU track for these alignments is searchable by gene name, accession number and PubMed identifier. Chromosome-level visualization of this track shows that the genes studied so far are distributed evenly on each chromosome, except for the repeatrich YSR (Fig. 8). In line with the observed loss of synteny in the Hox genes noted in Oikopleura [40], we did not see apparent clustering of genes by function or relatedness. The view of the OKI2018_I69 genome assembly can be found here:

https://fantom.gsc.riken.jp/zenbu/gLyphs/\#config= 0tPT7vwSO1Vm5QV9iKqfAC;loc=OKI2018_I69_1.0:: chr1:677717..880998+ (ZENBU view "OKI2018_I69_1.0 view with tracks (updated)").

\section{Conclusions}

We demonstrated that a combination of long- and short-read sequencing data from a single animal, together with the long-range $\mathrm{Hi}-\mathrm{C}$ data and the use of various bioinformatic approaches can result in a highquality de novo chromosome-scale assembly of $O$. dioica's highly polymorphic genome. However, further work is needed to properly resolve the polymorphisms into separated haplotypes using a different approach, such as trio-binning. We believe that the current version of the assembly will serve as an essential resource for a broad range of biological studies, including genomewide comparative studies of Oikopleura and other species, and provides insights into chromosomal evolution.

\section{Methods}

\section{Oikopleura sample and culture}

Wild live specimens were collected from Ishikawa Harbor $\left(26^{\circ} 25^{\prime} 39.3^{\prime \prime} \mathrm{N} 127^{\circ} 49^{\prime} 56.6^{\prime \prime} \mathrm{E}\right)$ by a hand-held plankton net and returned to the lab for culturing [5]. A typical generation time from hatchling to fully mature adult is 4 days at $23^{\circ} \mathrm{C}$ for the Okinawan $O$. dioica. Individuals I 28 and I69 were collected at generation 44 and 47 , respectively.

\section{Isolation and sequencing of DNA}

Staged fully mature males were collected prior to spawning. Each male was washed with $5 \mathrm{ml}$ filtered autoclaved seawater (FASW) for $10 \mathrm{~min}$ three times before resuspension in $50 \mu \mathrm{l} 4 \mathrm{M}$ guanidium isothiocyanate, $0.5 \%$ SDS, $50 \mathrm{mM}$ sodium citrate and $0.05 \% \quad \mathrm{v} / \mathrm{v} \quad 2-$ mercaptoethanol. This was left on ice for $30 \mathrm{~min}$ before being precipitated with 2 volumes of ice-cold ethanol and centrifuged at $14,000 \mathrm{rpm} 4{ }^{\circ} \mathrm{C}$ for $20 \mathrm{~min}$. The pellet 


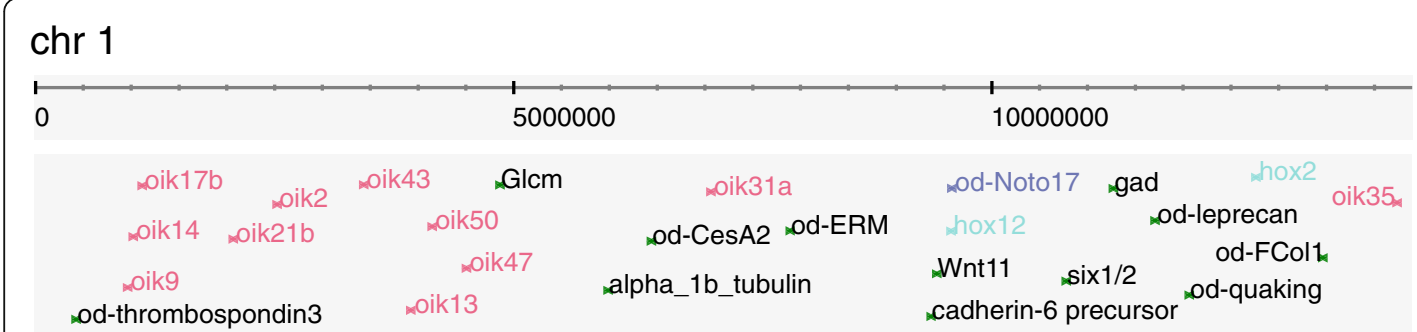

$\operatorname{chr} 2$

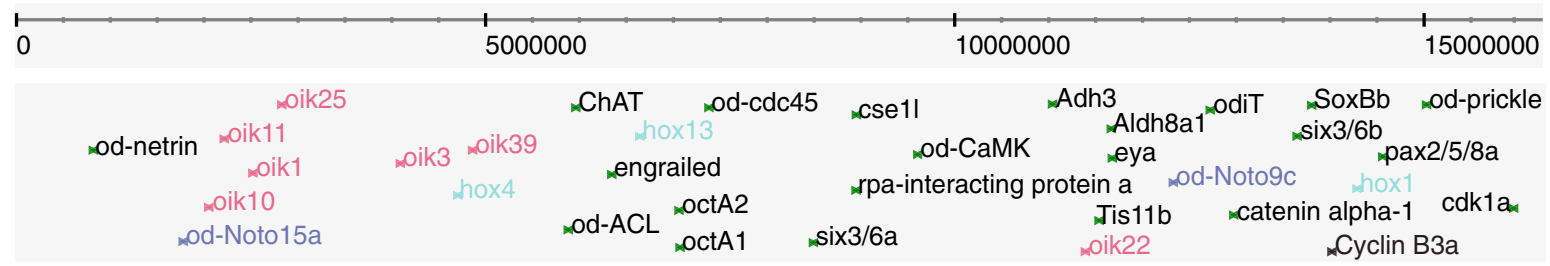

\section{PAR}

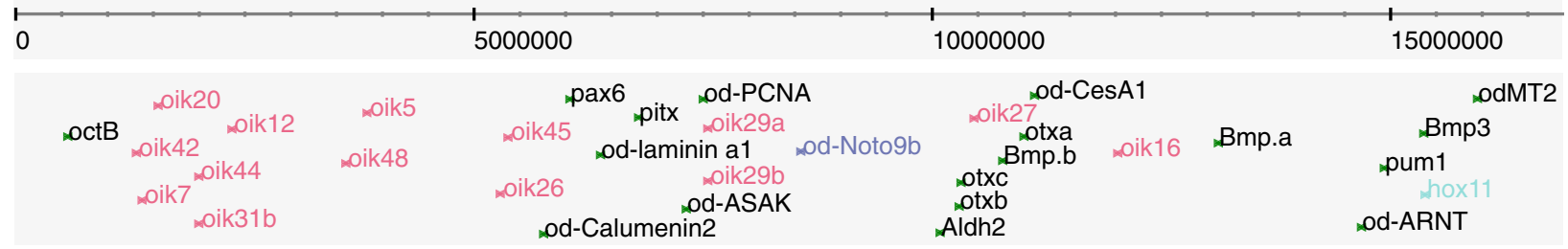

\section{XSR}

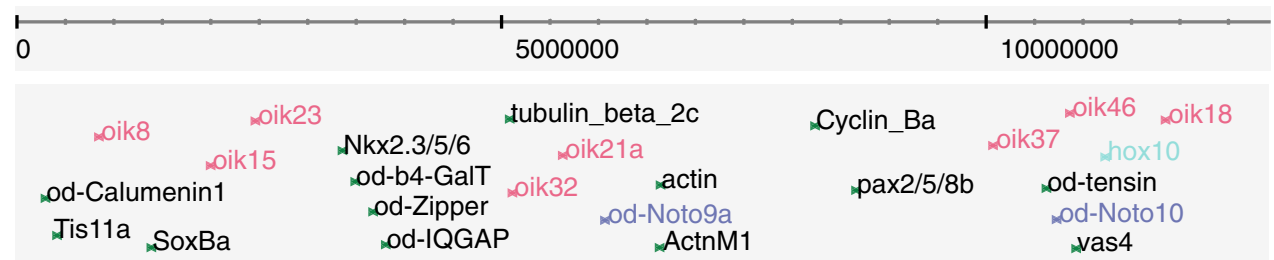

\section{YSR}

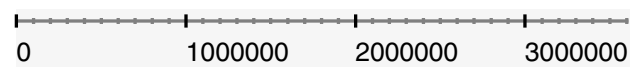

Fig. 8 Genomic locations of various oikopleurid gene homologs in the OKI2018_169. The genes are searchable by name and PubMed identifiers in the ZENBU genome browser. Colours indicate genes from the same family

was washed with $1 \mathrm{ml}$ of $70 \%$ cold-ethanol, centrifuged at $14,000 \mathrm{rpm} 4{ }^{\circ} \mathrm{C}$ for $5 \mathrm{~min}$ and air dried briefly before resuspension in $200 \mu \mathrm{l} 100 \mathrm{mM} \mathrm{NaCl}, 25 \mathrm{mM}$ EDTA, $0.5 \%$ SDS and $10 \mu \mathrm{g} / \mathrm{ml}$ proteinase $\mathrm{K}$. The lysates were incubated overnight at $50^{\circ} \mathrm{C}$. The next morning, the total nucleic acids were first extracted and then backextracted once more with chloroform:phenol (1:1). Organic and aqueous phases were resolved by centrifugation at 13,000 rpm for $5 \mathrm{~min}$ for each extraction; both first and back-extracted aqueous phases were collected and pooled. The pooled aqueous phase was subjected to a final extraction with chloroform and spun down as previously described. The aqueous fraction was then removed and precipitated by centrifugation with two volumes of cold ethanol and $10 \mu \mathrm{g} / \mathrm{ml}$ glycogen; washed with $1 \mathrm{ml}$ of cold $70 \%$ ethanol and centrifuged once more as previously described. The resulting pellet was allowed to air-dry for $5 \mathrm{~min}$ and finally resuspended in molecular biology grade $\mathrm{H}_{2} \mathrm{O}$ for quantitation using a Qubit 3 Fluorometer (Thermo Fisher Scientific, 
Q32850), and the integrity of the genomic DNA was validated using Agilent 4200 TapeStation (Agilent, 50675365).

Isolated genomic DNA used for long-reads on Nanopore MinION platform were processed with the Ligation Sequencing Kit (Nanopore LSK109) according to manufacturer's protocol, loading approximately $200 \mathrm{ng}$ total sample per R9.4 flow-cell. Raw signals were converted to sequence files with the Guppy proprietary software (model "template_r9.4.1_450bps_large_flipflop", version 2.3.5). Approximately $5 \mathrm{ng}$ was set aside for whole genome amplification to perform sequencing on Illumina MiSeq platform, using the TruePrime WGA Kit (Sygnis, $370,025)$ according to manufacturer's protocol. Magnetic bead purification (Promega, NG2001) was employed for all changes in buffer conditions required for enzymatic reactions and for final buffer suitable for sequencing system. Approximately $1 \mu \mathrm{g}$ of amplified DNA was sequenced by our core sequencing facility with a 600-cycle MiSeq Reagent Kit v3 (Illumina, MS-102-3003) following the manufacturer's instructions. These Illumina runs were used for polishing and error checking of Nanopore runs.

\section{Hi-C library preparation}

50 fully matured males were rinsed three times for 10 min each by transferring from well to well in a 6-well plate filled with $5 \mathrm{ml}$ FASW. Rinsed animals were combined in a $1.5 \mathrm{ml}$ microcentrifuge tube. Tissues were pelleted for $10 \mathrm{~min}$ at $12,000 \mathrm{rpm}$ and leftover FASW was discarded. A Hi-C library was then prepared by following the manufacturer's protocol (Dovetail, 21,004). Briefly, tissues were cross-linked for 20 min by adding 1

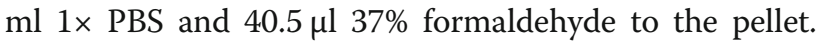
The tubes were kept rotating to avoid tissue settle during incubation. Cross-linked DNA was then blunt-end digested with DpnII (Dovetail) to prepare ends for ligation. After ligation, crosslinks were reversed, DNA was purified by AMPure XP Beads (Beckman, A63880) and quantified by Qubit 3 Fluorometer (Thermo Fisher Scientific, Q10210). The purified DNA was sheared to a size of $250-450$ bp by sonication using a Covaris M220 instrument (Covaris, Woburn, MA) with peak power 50 W, duty factor 20, and cycles/burst 200 times for $65 \mathrm{~s}$. DNA end repair, adapter ligation, PCR enrichment, and size selection were carried out by using reagents provided with the kit (Dovetail, 21,004). Finally, the library was checked for quality and quantity on an Agilent 4200 TapeStation (Agilent, 5067-5584) and a Qubit 3 Fluorometer. The library was sequenced on a MiSeq (Illumina, SY-410-1003) platform using a 300 cycles V2 sequencing kit (Illumina, MS-102-2002), yielding 20,832, 357 read pairs.

\section{Genome size estimation}

Jellyfish [41] was used to generate k-mer count profiles for various values of $k(17,21,25,29,33,37$, and 41) based on the genome-polishing Illumina MiSeq reads, with a maximum k-mer count of 1000. These k-mer profiles were subsequently used to estimate heterozygosity and genome size parameters using the GenomeScope web server [42].

\section{Filtering of Illumina MiSeq raw reads}

Before using at different steps, all raw Illumina reads were quality-filtered ( $-\mathrm{q} 30,-\mathrm{p} 70)$ and trimmed on both ends with the FASTX-Toolkit v0.0.14 [43]. The quality of the reads before and after filtering were checked with FASTQC v0.11.5 [44]. Read pairs that lacked one of the reads after the filtering were discarded in order to preserve paired-end information.

\section{Genome assembly}

Genome assembly was conducted with the Canu pipeline v1.8 [19] and $32.3 \mathrm{~Gb}(\sim 221.69 \times)$ raw Nanopore reads (correctedErrorRate $=0.105, \quad$ minReadLength $=1000$ ). The resulting contig assembly was polished three times with Racon v1.2.1 [45] using Canu-filtered Nanopore reads. Nanopore-specific errors were corrected with Pilon v1.22 [46] using filtered 150-bp paired-end Illumina reads $(\sim 99.7 \times)$. Illumina reads were aligned to the Canu contig assembly with BWA v0.7.17 [47] and the corresponding alignments were provided as input to Pilon. Next, one round of the HaploMerger2 processing pipeline [48] was applied to eliminate redundancy in contigs and to merge haplotypes.

Contigs were joined into scaffolds based on long-range Hi-C Dovetail ${ }^{\text {Tx }}$ data using Juicer v1.6 [49] and 3D de novo assembly (3D-DNA) [18] pipelines. The megabasescale scaffolds were joined into pairs of chromosome arms based on their synteny with the OdB3 physical map (see below). The candidate assembly was visualized and reviewed with Juicebox Assembly Tools (JBAT) v1.11.08 [50].

Whole-genome alignment between OKI2018_I69 and OdB3 assemblies was performed using LAST v1066 [51]. The sequence of OdB3 linkage groups were reconstructed as defined in the Supplementary Fig. 2 ("Draft chromosome scale assembly based on scaffolds of the reference genome sequence") in Denoeud et al. 2010 [9]. The resulting alignments were post-processed in $\mathrm{R}$ with a custom script (https://github.com/oist/ oikGenomePaper) and visualized using the $\mathrm{R}$ package "networkD3" ("sankeyNetwork" function). The color scheme for chromosomes was adopted from R Package RColourBrewer, "Set2".

The final assembly was checked for contamination by BLAST searches against the NCBI non-redundant 
sequence database. 12 smaller scaffolds were found to have strong matches to bacterial DNA (Suppl. Table 2), as well as possessing significantly higher Nanopore sequence coverage $(>500 x)$ than the rest of the assembly, and were therefore removed from the final assembly.

The completeness and quality of the assembly were checked with QUAST v5.0.2 [52] and by searching for the set of 978 highly conserved metazoan genes (OrthoDB version 9.1) [23] using BUSCO v3.0.2 [21, 22]. The --sp option was set to match custom AUGUSTUS parameters [24] trained using the Trinity transcriptome assembly (see below) split 50\% / 50\% for training and testing.

\section{Repeat masking and transposable elements}

A custom library of repetitive elements (RE) present in the genome assembly was built with RepeatModeler v2.0.1 that uses three de novo repeat finding programs: RECON v1.08, RepeatScout v1.0.6 and LtrHarvest/Ltr retriever v2.8. In addition, MITE-Hunter v11-2011 [53] and SINE_Finder [54] were used to search for MITE and SINE elements, respectively. The three libraries were pooled together as input to RepeatMasker v4.1.0 [55] to annotate and soft-mask these repeats in the genomic sequence. Resulting sets of REs were annotated by BLAST searches against RepeatMasker databases and sequences of transposable elements published for different oikopleurids [10].

Tandem repeats were detected using two different programs, tantan [56] and ULTRA [57] using two different maximal period lengths (100 and 2000). Version 23 of tantan was used with the parameters - $\mathrm{f} 4$ (output repeats) and -w100 or 2000 (maximum period length). ULTRA version 0.99 .17 was used with -mu 2 (minimum number of repeats) -p 100 or 2000 (maximum period length) and -mi 5 -md 5 (maximum consecutive insertions or deletions). ULTRA detected more tandem repeats than tantan, but its predictions include more than $90 \%$ of tantan's. Both tools detected O. dioica's telomeric tandem repeat sequence, which is TTAGGG as in other chordates [58].

\section{Developmental staging, isolation and sequencing of mRNA, transcriptome assembly}

Mixed stage embryos, immature adults (3 days after hatching) and adults (4 days after hatching) were collected separately from our on-going laboratory culture for RNA-Seq analysis. Eggs were washed three times for 10 min by moving eggs along with micropipette from well to well in a 6-well dish each containing $5 \mathrm{ml}$ of FASW and left in a fresh well of $5 \mathrm{ml} \mathrm{FASW}$ in the same dish. These were stored at $17^{\circ} \mathrm{C}$ and set aside for fertilization. Matured males, engorged with sperm, were also washed 3 times in FASW. Still intact mature males were placed in $100 \mu \mathrm{l}$ of fresh FASW and allowed to spawn naturally. Staged embryos were initiated by gently mixing $10 \mu \mathrm{l}$ of the spawned male sperm to the awaiting eggs in FASW at $23^{\circ} \mathrm{C}$. Generation 30 developing embryos at $1 \mathrm{~h}$ and $3 \mathrm{~h}$ post-fertilization were visually verified by dissecting microscope and collected as a pool for the mixed staged embryo time point. Immature adults at generation 31 and sexually differentiated adults at generation 30 were used for the two adult staged time points. All individuals for each time point were pooled and washed with FASW three times for $10 \mathrm{~min}$. Total RNA was extracted and isolated with RNeasy Micro Kit (Qiagen, 74,004) and quantitated using Qubit 3 Fluorometer (Thermo Fisher Scientific, Q10210). Additional quality control and integrity of isolated total RNA was checked using Agilent 4200 TapeStation (Agilent, 5067-5576). Further processing for mRNA selection was performed with Oligo-d(T)25 Magnetic Beads (NEB, E7490) and the integrity of the RNA was validated once more with Agilent 4200 TapeStation (Agilent, 5067-5579). Adapters for the creation of DNA libraries for the Illumina platform were added per manufacturer's guidance (NEB, E7805) as were unique indexed oligonucleotides (NEB, E7600) to each of the three staged samples. Each cDNA library was sequenced paired-end with a 300cycle MiSeq Reagent Kit v2 (Illumina, MS-102-2002) loaded at approximately $12 \mathrm{pM}$.

After quality assessment and data filtering (see Filtering of Illumina MiSeq raw reads), Illumina RNA-Seq reads were pooled together and de novo assembled with Trinity v2.8.2 [59]. Redundancy in the transcriptome assembly was removed by CD-HIT v4.8.1 [60] with a cutoff value of $95 \%$ identity. The quality and completeness of the transcriptome assembly was verified with rnaQUAST v1.5.1 [61] and BUSCO.

\section{Gene prediction and annotation}

Gene models were predicted using AUGUSTUS v3.3 [62]. AUGUSTUS was trained following the Hoff and Stanke protocol [24] with the initial RNA-Seq reads and transcriptome assembly used as intron and exon hints, correspondingly. Transcript models were generated with the PASA pipeline v20140417 [63] using BLAT v36 and GMAP v2018-02-12 to align transcripts to the genome. RNA-Seq reads were mapped to the genome with STAR v2.0.6a [64]. Running AUGUSTUS using hints resulted in a set of 17,277 protein-coding genes and 18,811 transcript models. Chromosomal coordinates were ported to our final assembly using the Liftoff tool [65] filtering out 17 genes and corresponding transcripts. The quality of the predicted gene models was assessed with BUSCO.

A draft annotation of the mitochondrial genome was obtained by submitting the corresponding scaffold ( $\mathrm{chr}_{-}$ Un12) as input to the MITOS2 mitochondrial genome 
annotation server [66] (accessed May 28, 2020) with the ascidian mitochondrial translation table specified $[9,32]$.

\section{Detection of coding RNAs}

A translated alignment was used to detect known $O$. dioica genes available from GenBank using the TBLASTN software [67] with the options -ungapped -comp_based_stats $\mathrm{F}$ to prevent $\mathrm{O}$. dioica's small introns from being incorporated as alignment gaps, and -max intron_length 100,000 to reflect the compactness of $O$. dioica's genome. The best hits were converted to GFF3 format using BioPerl's bp_search2gff program [68] before being uploaded to the ZENBU genome browser [39]. For some closely related pairs of genes that gave ambiguous results with that method, we searched for the protein sequence in our transcriptome assembly with TBLASTN, located the genomic region where the best transcript model hit was aligned, and selected the hit from the original TBLASTN search that matched this region. We summarized our results in Suppl. Table 6. For both searches, we used an $E$-value filter of $10^{-40}$. Genes marked as not found in the table might be present in the genome while failing to pass the filter.

\section{Detection of non-coding RNAs}

To validate the results of cmscan on rRNAs, genomic regions were screened with a nucleotide BLAST search using the $O$. dioica isolate MT01413 18S ribosomal RNA gene, partial sequence (GenBank:KJ193766.1). 200kbp windows surrounding the hits where then analysed with the RNAmmer 1.2 web service [69]. RNAmmer did not detect the 5.8S RNA, but we could confirm its presence by a nucleotide BLAST search using the AF158726.1 reference sequence. The loci containing the 5S rRNA (AJ628166) and the spliced leader RNA (AJ628166) were detected with the exonerate 2.4 software [70], with its affine:local model and a score threshold of 1000 using the region chr1:8487589-8,879,731 as a query.

\section{Whole-genome alignments}

Pairs of genomes were mapped to each other with the LAST software [51] version 1066. When indexing the reference genome, we replaced the original lowercase soft masks with ones for simple repeats (lastdb -R01) and we selected a scoring scheme for near-identical matches (-uNEAR). Substitution and gap frequencies were determined with last-train [71], with the alignment options -E0.05 -C2 and forcing symmetry with the options --revsym --matsym --gapsym. An optimal set of pairwise one-to-one alignments was then calculated using last-split [72]. For visualization of the results, we converted the alignments to GFF3 format and collated the colinear "match_part" alignment blocks in "match" regions using LAST's command maf-convert -J 200000. We then collated syntenic region blocks (sequence ontology term SO:0005858) that map to the same sequence landmark (chromosome, scaffold, contigs) on the query genome with a distance of less than $500,000 \mathrm{bp}$ with the custom script syntenic_regions.sh (https:// github.com/oist/oikGenomePaper). In contrast to the "match" regions, the syntenic ones are not necessarily colinear and can overlap with each other. The GFF3 file was then uploaded to the ZENBU genome browser.

\section{Nanopore read realignments}

Nanopore reads were realigned to the genome with the LAST software [51] as in the whole-genome alignments above. FASTQ qualities were discarded with the option -Q0 of lastal. Optimal split alignments were calculated with last-split. Alignment blocks belonging to the same read were joined with maf-convert - $\mathrm{J} 1 \mathrm{e} 6$ and the custom script syntenic_regions_stranded.sh. The resulting GFF3 files were loaded in the ZENBU genome browser to visualize the alignments near gap regions in order to check for reads spanning the gaps.

\section{Analysis of sequence properties across chromosome-scale scaffolds}

Each chromosome-scale scaffold was separated into windows of $50 \mathrm{kbp}$ and evaluated for GC content, repeat content, sequencing depth, and the presence of DpnII restriction sites. For chr 1, chr 2, and the PAR, windows corresponding to long and short chromosome arms were separated based on their positioning relative to a central gap region (chr 1 short arm: 1-5,191,657 bp, chr 1 long arm: 5,192,156-14,533,022 bp; chr 2 short arm: 1-5,707, 009, chr 2 long arm: 5,707,508-16,158,756 bp; PAR short arm: 1-6,029,625 bp, PAR long arm: 6,030,124-17,092, 476). Since none of our assemblies or sequencing reads spanned both the PAR and either sex-specific chromosome, the $\mathrm{X}$ and $\mathrm{Y}$ chromosomes were excluded from this analysis. For each of GC content, sequencing depth, repeat content, gene count, and DpnII restriction sites, the significance of the differences between long and short arms was assessed with Welch's two-sided T test as well as a nonparametric Mann-Whitney test implemented in $\mathrm{R}$ (Suppl. Table 3). The results of the two tests were largely in agreement, but groups were only indicated as significantly different if they both produced significance values below $0.05(p<0.05)$.

\section{Abbreviations}

BUSCOs: Benchmarking Universal Single-Copy Orthologs; chr 1: Autosomal chromosome 1; chr 2: Autosomal chromosome 2; kbp: Kilobase pairs; LGs: Linkage groups; Mbp: Megabase pairs; PAR: Pseudo-autosomal regions; XSR: X-specific region; YSR: Y-specific region 


\section{Supplementary Information}

The online version contains supplementary material available at https://doi. org/10.1186/s12864-021-07512-6.

Additional file 1: Table S1. Per-scaffold statistics. Table S2. Contaminations found in smaller scaffold of the OKI2018_169 assembly. Table S3. Statistics results for the analysis of sequence properties across chromosome-scale scaffolds. Table S4. BUSCO scores. Table S5. List of missing BUSCO genes in OKI2018_169, OdB3 and OSKA2016 genome assemblies. Table S6. List of oikopleurid gene homologs uploaded to ZENBU.

\section{Acknowledgements}

We would like to thank the DNA Sequencing Section and the Scientific Computing and Data Analysis Section of the Research Support Division at OIST for their support, Danny Miller for advices on Nanopore sequencing, Dan Rokhsar, Gene Myers, Ferdinand Marlétaz and Konstantin Khalturin for critical comments, Takeshi Onuma and Hiroki Nishida for sharing the OSKA2016 genome sequence prior publication, Simon Henriet for the technical advice on the DNA extraction protocol, and Lin Zhang for helping with the culture.

\section{Authors' contributions}

Conceptualization: $A B, C P, N M L$; data curation: $A B, M J M, C P$; formal analysis: $A B, M J M, C W, T R, H C C, S K, J P, C P$; investigation: $A B, A M, M J M, Y K T, A W L, C P$; methodology: $A B, C P$; project administration: $A B, C P$; software: $M J M, C W$; supervision: $C P, N M L$; validation: $A B$; visualization: $A B, A M, M J M, Y K T$; writing - original draft: $A B, A M, M J M, Y K T$, CP; writing - review \& editing: $A B, M J M$, $C P, N M L$. All authors read and approved the final manuscript.

\section{Funding}

MJM received funding as an International Research Fellow of the Japan Society for the Promotion of Science. This work was supported by core funding from the Okinawa Institute of Science and Technology Graduate University.

\section{Availability of data and materials}

All sequence data presented here, the final OKI2018_169_1.0 genome assembly and annotation were deposited to the ENA database under BioProject ID PRJEB40135 and Zenodo (DOI https://doi.org/10.5281/zenodo. 4604144). Custom scripts used in this study are available in GitHub (https:// github.com/oist/oikGenomePaper).

\section{Declarations}

\section{Ethics approval and consent to participate}

Not applicable.

\section{Consent for publication}

Not applicable.

\section{Competing interests}

The authors declare that they have no competing interests.

\section{Author details}

${ }^{1}$ Genomics and Regulatory Systems Unit, Okinawa Institute of Science and Technology Graduate University, Okinawa, Japan. ${ }^{2}$ Francis Crick Institute, London, UK. ${ }^{3}$ Department of Genetics, Evolution and Environment, UCL Genetics Institute, University College London, London, UK.

Received: 21 September 2020 Accepted: 5 March 2021

Published online: 29 March 2021

\section{References}

1. Alldredge AL. Discarded appendicularian houses as sources of food, surface habitats, and particulate organic matter in planktonic environments. Limnol Oceanogr. 1976;21(1):14-24. https://doi.org/10.4319/lo.1976.21.1.0014.

2. Hopcroft RR, Roff JC. Zooplankton growth rates: extraordinary production by the larvacean Oikopleura dioica in tropical waters. J Plankton Res. 1995: 17(2):205-20. https://doi.org/10.1093/plankt/17.2.205.
3. Sato R, Tanaka Y, Ishimaru T. House production by Oikopleura dioica (Tunicata, Appendicularia) under laboratory conditions. J Plankton Res. 2001; 23(4):415-23. https://doi.org/10.1093/plankt/23.4.415.

4. Alldredge $\mathrm{A}$. The contribution of discarded appendicularian houses to the flux of particulate organic carbon from oceanic surface waters. In: Gorsky G, Youngbluth MJ, Deibel D, editors. Response of Marine Ecosystems to Global Change: Ecological Impact of Appendicularians: Contemporaty Publishing International; 2005. p. 309-26.

5. Masunaga A, Liu AW, Tan Y, Scott A, Luscombe NM. Streamlined sampling and cultivation of the pelagic cosmopolitan larvacean, Oikopleura dioica. JoVE (Journal of Visualized Experiments). 2020;16(160):e61279.

6. Fenaux R. Anatomy and functional morphology of the Appendicularia. In: Bone Q, editor. The biology of pelagic tunicates: Oxford University Press; 1998. p. 25-34.

7. Delsuc F, Brinkmann $H$, Chourrout D, Philippe $H$. Tunicates and not cephalochordates are the closest living relatives of vertebrates. Nature. 2006 439(7079):965-8. https://doi.org/10.1038/nature04336.

8. Seo HC, Kube M, Edvardsen RB, Jensen MF, Beck A, Spriet E, Gorsky G, Thompson EM, Lehrach H, Reinhardt R, Chourrout D. Miniature genome in the marine chordate Oikopleura dioica. Science. 2001;294(5551):2506. https:// doi.org/10.1126/science.294.5551.2506.

9. Denoeud F, Henriet S, Mungpakdee S, Aury JM, Da Silva C, Brinkmann H, Mikhaleva J, Olsen LC, Jubin C, Cañestro C, Bouquet JM. Plasticity of animal genome architecture unmasked by rapid evolution of a pelagic tunicate. Science. 2010;330(6009):1381-5. https://doi.org/10.1126/science.1194167.

10. Naville M, Henriet S, Warren I, Sumic S, Reeve M, Volff JN, Chourrout D. Massive changes of genome size driven by expansions of non-autonomous transposable elements. Curr Biol. 2019;29(7):1161-8. https://doi.org/10.1016/ j.cub.2019.01.080.

11. Fredriksson G, Olsson R. The subchordal cells of Oikopleura dioica and O. albicans (Appendicularia, Chordata). Acta Zool. 1991;72(4):251-6. https://doi. org/10.1111/j.1463-6395.1991.tb01203.x

12. Wang K, Omotezako T, Kishi K, Nishida H, Onuma TA. Maternal and zygotic transcriptomes in the appendicularian, Oikopleura dioica: novel proteinencoding genes, intra-species sequence variations, and trans-spliced RNA leader. Dev Genes Evol. 2015;225(3):149-59. https://doi.org/10.1007/s00427015-0502-7.

13. Wang K, Tomura $\mathrm{R}$, Chen W, Kiyooka M, Ishizaki H, Aizu T, Minakuchi Y, Seki M, Suzuki Y, Omotezako T, Suyama R. A genome database for a Japanese population of the larvacean Oikopleura dioica. Develop Growth Differ. 2020; 62(6):450-61. https://doi.org/10.1111/dgd.12689.

14. Körner WF. Untersuchungen über die gehäusebildung bei appendicularien (Oikopleura dioica fol). Z Morphol Okol Tiere. 1952;41(1):1-53. https://doi. org/10.1007/BF00407623.

15. Colombera D, Fenaux R. Chromosome form and number in the Larvacea. Ital J Zool. 1973;40(3-4):347-53

16. Liu AW, Tan Y, Masunaga A, Bliznina A, West C, Plessy C, Luscombe NM. H3S28P Antibody Staining of Okinawan Oikopleura dioica Suggests the Presence of Three Chromosomes. F1000Research. 2021; 9:780. https://doi. org/10.12688/f1000research.25019.2.

17. Lieberman-Aiden $\mathrm{E}_{1}$ Van Berkum NL, Williams L, Imakaev M, Ragoczy T, Telling A, Amit I, Lajoie BR, Sabo PJ, Dorschner MO, Sandstrom R. Comprehensive mapping of long-range interactions reveals folding principles of the human genome. Science. 2009;326(5950):289-93. https:// doi.org/10.1126/science.1181369.

18. Dudchenko O, Batra SS, Omer AD, Nyquist SK, Hoeger M, Durand NC, Shamim MS, Machol I, Lander ES, Aiden AP, Aiden EL. De novo assembly of the Aedes aegypti genome using hi-C yields chromosome-length scaffolds. Science. 2017;356(6333):92-5. https://doi.org/10.1126/science.aal3327.

19. Koren S, Walenz BP, Berlin K, Miller JR, Bergman NH, Phillippy AM. Canu: scalable and accurate long-read assembly via adaptive k-mer weighting and repeat separation. Genome Res. 2017;27(5):722-36. https://doi.org/10.1101/ gr.215087.116

20. Melters DP, Bradnam KR, Young HA, Telis N, May MR, Ruby JG, Sebra R, Peluso P, Eid J, Rank D, Garcia JF. Comparative analysis of tandem repeats from hundreds of species reveals unique insights into centromere evolution. Genome Biol. 2013;14(1):1-20.

21. Simão FA, Waterhouse RM, loannidis P, Kriventseva EV, Zdobnov EM. BUSCO: assessing genome assembly and annotation completeness with single-copy orthologs. Bioinformatics. 2015;31(19):3210-2. https://doi.org/1 0.1093/bioinformatics/btv351. 
22. Waterhouse RM, Seppey M, Simão FA, Manni M, loannidis P, Klioutchnikov G, Kriventseva EV, Zdobnov EM. BUSCO applications from quality assessments to gene prediction and phylogenomics. Mol Biol Evol. 2018; 35(3):543-8. https://doi.org/10.1093/molbev/msx319.

23. Zdobnov EM, Tegenfeldt F, Kuznetsov D, Waterhouse RM, Simao FA, loannidis P, Seppey M, Loetscher A, Kriventseva EV. OrthoDB v9. 1: cataloging evolutionary and functional annotations for animal, fungal, plant, archaeal, bacterial and viral orthologs. Nucleic Acids Res. 2017;45(D1):D7449. https://doi.org/10.1093/nar/gkw1119.

24. Hoff KJ, Stanke M. Predicting genes in single genomes with augustus. Curr Protoc Bioinformatics. 2019;65(1):e57. https://doi.org/10.1002/cpbi.57.

25. Satou Y, Nakamura R, Yu D, Yoshida R, Hamada M, Fujie M, Hisata K, Takeda $\mathrm{H}$, Satoh N. A nearly complete genome of Ciona intestinalis type a ( $C$. robusta) reveals the contribution of inversion to chromosomal evolution in the genus Ciona. Genome Biol Evol. 2019;11(11):3144-57. https://doi.org/1 0.1093/gbe/evz228.

26. Blanchoud S, Rutherford K, Zondag L, Gemmell NJ, Wilson MJ. De novo draft assembly of the Botrylloides leachii genome provides further insight into tunicate evolution. Sci Rep. 2018;8(1):1-8.

27. Žárský $\vee$, Tachezy J. Evolutionary loss of peroxisomes-not limited to parasites. Biol Direct. 2015;10(1):1-0.

28. Kienle N, Kloepper TH, Fasshauer D. Shedding light on the expansion and diversification of the Cdc48 protein family during the rise of the eukaryotic cell. BMC Evol Biol. 2016;16(1):215. https://doi.org/10.1186/s12862-0160790-1.

29. Berná L, D'Onofrio G, Alvarez-Valin F. Peculiar patterns of amino acid substitution and conservation in the fast evolving tunicate Oikopleura dioica. Mol Phylogenet Evol. 2012;62(2):708-17. https://doi.org/10.1016/j.ympev.2 011.11.013.

30. Berná L, Alvarez-Valin F. Evolutionary volatile Cysteines and protein disorder in the fast evolving tunicate Oikopleura dioica. Mar Genomics. 2015;24:47-54.

31. Ganot P, Kallesøe T, Reinhardt R, Chourrout D, Thompson EM. Spliced-leader RNA trans splicing in a chordate, Oikopleura dioica, with a compact genome. Mol Cell Biol. 2004;24(17):7795-805. https://doi.org/10.1128/ MCB.24.17.7795-7805.2004.

32. Pichon J, Luscombe NM, Plessy C. Widespread use of the "ascidian" mitochondrial genetic code in tunicates. F1000Research. 2019;8.

33. Kolmogorov M, Yuan J, Lin Y, Pevzner PA. Assembly of long, error-prone reads using repeat graphs. Nat Biotechnol. 2019;37(5):540-6. https://doi. org/10.1038/s41587-019-0072-8.

34. Drosophila 12 Genomes Consortium, et al. Nature. 2007;450(7167):203.

35. Simakov O, Marlétaz F, Yue JX, O'Connell B, Jenkins J, Brandt A, Calef R, Tung CH, Huang TK, Schmutz J, Satoh N. Deeply conserved synteny resolves early events in vertebrate evolution. Nat Ecol Evol. 2020;20:1-11.

36. Schaeffer SW. Muller "elements" in Drosophila: how the search for the genetic basis for speciation led to the birth of comparative genomics. Genetics. 2018;210(1):3-13. https://doi.org/10.1534/genetics.118.301084.

37. Shoguchi E, Kawashima T, Nishida-Umehara C, Matsuda Y, Satoh N. Molecular cytogenetic characterization of Ciona intestinalis chromosomes. Zool Sci. 2005;22(5):511-6. https://doi.org/10.2108/zsj.22.511.

38. Ganot $P$, Thompson EM. Patterning through differential endoreduplication in epithelial organogenesis of the chordate, Oikopleura dioica. Dev Biol. 2002;252(1):59-71. https://doi.org/10.1006/dbio.2002.0834.

39. Severin J, Lizio M, Harshbarger J, Kawaji H, Daub CO, Hayashizaki Y, Bertin N, Forrest AR. Interactive visualization and analysis of large-scale sequencing datasets using ZENBU. Nat Biotechnol. 2014;32(3):217-9. https://doi.org/10.1 038/nbt.2840

40. Seo HC, Edvardsen RB, Maeland AD, Bjordal M, Jensen MF, Hansen A, Flaat $M$, Weissenbach J, Lehrach H, Wincker P, Reinhardt R. Hox cluster disintegration with persistent anteroposterior order of expression in Oikopleura dioica. Nature. 2004;431(7004):67-71. https://doi.org/10.1038/na ture02709.

41. Marçais G, Kingsford C. A fast, lock-free approach for efficient parallel counting of occurrences of k-mers. Bioinformatics. 2011;27(6):764-70. https://doi.org/10.1093/bioinformatics/btr011.

42. Vurture GW, Sedlazeck FJ, Nattestad M, Underwood CJ, Fang H, Gurtowski J, Schatz MC. GenomeScope: fast reference-free genome profiling from short reads. Bioinformatics. 2017;33(14):2202-4. https://doi.org/10.1093/bioinforma tics/btx153.

43. Gordon A, Hannon GJ. Fastx-toolkit. FASTQ/A short-reads preprocessing tools (unpublished) http://hannonlab.cshl.edu/fastx_toolkit/. 2010;5.
44. Andrews S. FastQC: a quality control tool for high throughput sequence data; 2010.

45. Vaser R, Sović I, Nagarajan N, Šikić M. Fast and accurate de novo genome assembly from long uncorrected reads. Genome Res. 2017;27(5):737-46. https://doi.org/10.1101/gr.214270.116.

46. Walker BJ, Abeel T, Shea T, Priest M, Abouelliel A, Sakthikumar S, Cuomo CA, Zeng Q, Wortman J, Young SK, Earl AM. Pilon: an integrated tool for comprehensive microbial variant detection and genome assembly improvement. PLoS One. 2014;9(11):e112963. https://doi.org/10.1371/journal. pone.0112963.

47. Li H. Aligning sequence reads, clone sequences and assembly contigs with BWA-MEM. arXiv preprint arXiv. 2013;1303:3997.

48. Huang S, Kang M, Xu A. HaploMerger2: rebuilding both haploid subassemblies from high-heterozygosity diploid genome assembly. Bioinformatics. 2017;33(16):2577-9. https://doi.org/10.1093/bioinformatics/ btx220.

49. Durand NC, Shamim MS, Machol I, Rao SS, Huntley MH, Lander ES, Aiden EL. Juicer provides a one-click system for analyzing loop-resolution hi-C experiments. Cell Syst. 2016;3(1):95-8. https://doi.org/10.1016/j.cels.2016.07. 002.

50. Durand NC, Robinson JT, Shamim MS, Machol I, Mesirov JP, Lander ES, Aiden EL. Juicebox provides a visualization system for hi-C contact maps with unlimited zoom. Cell Syst. 2016;3(1):99-101. https://doi.org/10.1016/j. cels.2015.07.012.

51. Kiełbasa SM, Wan R, Sato K, Horton P, Frith MC. Adaptive seeds tame genomic sequence comparison. Genome Res. 2011;21(3):487-93. https://doi. org/10.1101/gr.113985.110.

52. Gurevich A, Saveliev V, Vyahhi N, Tesler G. QUAST: quality assessment tool for genome assemblies. Bioinformatics. 2013;29(8):1072-5. https://doi.org/1 0.1093/bioinformatics/btt086.

53. Han Y, Wessler SR. MITE-hunter: a program for discovering miniature inverted-repeat transposable elements from genomic sequences. Nucleic Acids Res. 2010;38(22):e199. https://doi.org/10.1093/nar/gkq862.

54. Wenke T, Döbel T, Sörensen TR, Junghans H, Weisshaar B, Schmidt T. Targeted identification of short interspersed nuclear element families shows their widespread existence and extreme heterogeneity in plant genomes. Plant Cell. 2011;23(9):3117-28. https://doi.org/10.1105/tpc.111.088682.

55. Smit A.F.A., Hubley R. \& Green P. RepeatMasker at http://repeatmasker.org

56. Frith MC. A new repeat-masking method enables specific detection of homologous sequences. Nucleic Acids Res. 2011;39(4):e23. https://doi.org/1 $0.1093 /$ nar/gkq1212.

57. Olson D, Wheeler T. ULTRA: A Model Based Tool to Detect Tandem Repeats. In: Proceedings of the 2018 ACM International Conference on Bioinformatics, Computational Biology, and Health Informatics; 2018. p. 37-46.

58. Schulmeister A, Schmid M, Thompson EM. Phosphorylation of the histone H3. 3 variant in mitosis and meiosis of the urochordate Oikopleura dioica. Chromosom Res. 2007;15(2):189.

59. Grabherr MG, Haas BJ, Yassour M, Levin JZ, Thompson DA, Amit I, Adiconis $X$, Fan L, Raychowdhury R, Zeng Q, Chen Z. Full-length transcriptome assembly from RNA-Seq data without a reference genome. Nat Biotechnol. 2011;29(7):644-52. https://doi.org/10.1038/nbt.1883.

60. Li W, Godzik A. Cd-hit: a fast program for clustering and comparing large sets of protein or nucleotide sequences. Bioinformatics. 2006;22(13):1658-9. https://doi.org/10.1093/bioinformatics/btl158.

61. Bushmanova E, Antipov D, Lapidus A, Suvorov V, Prjibelski AD. rnaQUAST: a quality assessment tool for de novo transcriptome assemblies. Bioinformatics. 2016;32(14):2210-2. https://doi.org/10.1093/bioinformatics/ btw218.

62. Stanke M, Schöffmann O, Morgenstern B, Waack S. Gene prediction in eukaryotes with a generalized hidden Markov model that uses hints from external sources. BMC Bioinformatics. 2006;7(1):62. https://doi.org/10.1186/14 71-2105-7-62.

63. Haas BJ, Delcher AL, Mount SM, Wortman JR, Smith RK Jr, Hannick LI, Maiti R, Ronning CM, Rusch DB, Town CD, Salzberg SL. Improving the Arabidopsis genome annotation using maximal transcript alignment assemblies. Nucleic Acids Res. 2003;31(19):5654-66. https://doi.org/10.1093/nar/gkg770.

64. Dobin A, Davis CA, Schlesinger F, Drenkow J, Zaleski C, Jha S, Batut P, Chaisson M, Gingeras TR. STAR: ultrafast universal RNA-seq aligner. Bioinformatics. 2013;29(1):15-21. https://doi.org/10.1093/bioinformatics/ bts635. 
65. Shumate A, Salzberg S. Liftoff: an accurate gene annotation mapping tool. bioRxiv. 2020. https://doi.org/10.1101/2020.06.24.169680.

66. Bernt M, Donath A, Jühling F, Externbrink F, Florentz C, Fritzsch G, Pütz J, Middendorf M, Stadler PF. MITOS: improved de novo metazoan

mitochondrial genome annotation. Mol Phylogenet Evol. 2013;69(2):313-9. https://doi.org/10.1016/j.ympev.2012.08.023.

67. Gertz EM, Yu YK, Agarwala R, Schäffer AA, Altschul SF. Composition-based statistics and translated nucleotide searches: improving the TBLASTN module of BLAST. BMC Biol. 2006;4(1):1-4.

68. Stajich JE, Block D, Boulez K, Brenner SE, Chervitz SA, Dagdigian C, Fuellen G, Gilbert JG, Korf I, Lapp H, Lehväslaiho H. The Bioperl toolkit: Perl modules for the life sciences. Genome Res. 2002;12(10):1611-8. https://doi.org/10.11 01/gr.361602

69. Lagesen K, Hallin P, Rødland EA, Stærfeldt HH, Rognes T, Ussery DW. RNAmmer: consistent and rapid annotation of ribosomal RNA genes. Nucleic Acids Res. 2007;35(9):3100-8. https://doi.org/10.1093/nar/gkm160.

70. Slater GS, Birney E. Automated generation of heuristics for biological sequence comparison. BMC Bioinformatics. 2005;6(1):31. https://doi.org/1 0.1186/1471-2105-6-31.

71. Hamada M, Ono Y, Asai K, Frith MC. Training alignment parameters for arbitrary sequencers with LAST-TRAIN. Bioinformatics. 2017;33(6):926-8 https://doi.org/10.1093/bioinformatics/btw742.

72. Frith MC, Kawaguchi R. Split-alignment of genomes finds orthologies more accurately. Genome Biol. 2015;16(1):106. https://doi.org/10.1186/s13059-0150670-9.

\section{Publisher's Note}

Springer Nature remains neutral with regard to jurisdictional claims in published maps and institutional affiliations.

Ready to submit your research? Choose BMC and benefit from:

- fast, convenient online submission

- thorough peer review by experienced researchers in your field

- rapid publication on acceptance

- support for research data, including large and complex data types

- gold Open Access which fosters wider collaboration and increased citations

- maximum visibility for your research: over $100 \mathrm{M}$ website views per year

At $\mathrm{BMC}$, research is always in progress.

Learn more biomedcentral.com/submissions 